(1)

CrossMark

\title{
The clinical significance of oropharyngeal cultures in young children with cystic fibrosis
}

\author{
Oded Breuer (1) ${ }^{1,2,9}$, Daan Caudri ${ }^{1,2,3,9}$, Lauren Akesson ${ }^{1,4,5}$, \\ Sarath Ranganathan ${ }^{4,5,6,7}$, Stephen M. Stick ${ }^{1,2}$ and André Schultz ${ }^{1,2,8}$ \\ on behalf of AREST CF
}

Affiliations: ${ }^{1}$ Telethon Kids Institute, University of Western Australia, Perth, Australia. ${ }^{2}$ Princess Margaret Hospital for Children, Perth, Australia. ${ }^{3}$ Dept of Pediatrics/Respiratory Medicine, Erasmus MC, Rotterdam, The Netherlands. ${ }^{4}$ Dept of Paediatrics, University of Melbourne, Melbourne, Australia. ${ }^{5}$ Dept of General Medicine, Royal Children's Hospital, Melbourne, Australia. 'Murdoch Children's Research Institute, Parkville, Australia. ${ }^{7}$ Dept of Respiratory and Sleep Medicine, Royal Children's Hospital, Parkville, Australia. ${ }^{8}$ School of Paediatric and Child Health, University of Western Australia, Perth, Australia. ${ }^{9}$ These authors contributed equally to this work.

Correspondence: Oded Breuer, Telethon Kids Institute, P0 Box 855, West Perth, Western Australia 6872, Australia. E-mail: oded.breuerQtelethonkids.org.au

@ERSpublications

Oropharyngeal swab cultures in children with CF are not helpful for ruling out lower airway Pseudomonas infection and are not associated with structural lung disease, lung inflammation or admissions for respiratory exacerbations http://ow.ly/jAgM30jm2P4

Cite this article as: Breuer $\mathrm{O}$, Caudri $\mathrm{D}$, Akesson $\mathrm{L}$, et al. The clinical significance of oropharyngeal cultures in young children with cystic fibrosis. Eur Respir J 2018; 51: 1800238 [https://doi.org/10.1183/ 13993003.00238-2018].

ABSTRACT In children with cystic fibrosis (CF) the associations between oropharyngeal swabs (OPSs) for detection of Pseudomonas and lung disease have not been evaluated.

OPS and bronchoalveolar lavage (BAL) samples were obtained annually in children with CF from 2005 to 2017. OPS test characteristics were calculated using BAL as "gold standard". Results were related to lung inflammation (BAL neutrophil elastase and interleukin-8), structural lung disease (chest computed tomography PRAGMA-CF (Perth-Rotterdam Annotated Grid Morphometric Analysis for CF) scores), respiratory exacerbations and future detection of Pseudomonas on BAL.

From 181 patients, 690 paired OPS-BAL cultures were obtained. Prevalence of Pseudomonas in BAL was $7.4 \%$. OPS sensitivity was $23.0 \%$ and specificity was $91.4 \%$, reducing the post-test probability for a positive BAL following a negative OPS to $6.3 \%$. Pseudomonas on OPS was not associated with lung inflammation or respiratory exacerbations, but was weakly associated with current PRAGMA-CF \%Disease score $(\mathrm{p}=0.043)$. Pseudomonas on BAL was associated with positive neutrophil elastase (OR 4.17, 95\% CI 2.04-8.53; $\mathrm{p}<0.001)$, increased interleukin-8 $(\mathrm{p}<0.001)$, increased all baseline PRAGMA computed tomography scores $(\mathrm{p}<0.001)$, progression of PRAGMA computed tomography scores $(\mathrm{p}<0.05)$ and increased risk of respiratory exacerbations (incidence rate ratio $2.11,95 \%$ CI 1.15-3.87; $\mathrm{p}=0.017$ ).

In children with CF OPSs only marginally change the probability of detecting lower airway Pseudomonas and are not associated with lung disease indices nor exacerbations risk. 


\section{Introduction}

In infants and young children with cystic fibrosis (CF) the presence of pathogenic organisms, including Pseudomonas aeruginosa, in the lower airways is associated with increased inflammation [1-5], and later in childhood with structural lung disease and lower lung function [6-8]. International guidelines presently recommend regular upper airway cultures in infants and young children who are too young to expectorate in order to make management decisions to treat newly acquired as well as chronic $P$. aeruginosa infection [9-11]. For the majority of infants and young children, the collection of oropharyngeal swabs (OPSs) is the main sampling technique used for bacterial surveillance purposes. The perceived value of OPSs is related to previous reports of high specificity and negative predictive values [12-15], but the low sensitivity and the poor predictive accuracy of OPSs to reflect lower airway infection brings the value of OPSs into question [16]. Furthermore, previous studies that report the predictive value of OPSs were published prior to established newborn screening and aggressive early antipseudomonal regimens, which are likely to have reduced lower airway $P$. aeruginosa infection in young children with $\mathrm{CF}$ and, thus potentially, the predictive accuracy of OPSs $[4,17,18]$. Despite recognised limitations, the standard use of OPSs for bacterial surveillance and strong recommendation to treat $P$. aeruginosa when identified in young asymptomatic children with CF have led many centres to prescribe antipseudomonal treatment based upon OPS culture results. Determining therapy according to OPS culture may potentially result in delayed therapy in the case of false-negative results or in administration of unnecessary treatment for false-positive results. The aim of this study was to evaluate the associations between $P$. aeruginosa OPS cultures and current and future lower airway inflammation, structural lung disease, respiratory exacerbations, and lower airway $P$ aeruginosa infection in young children with $\mathrm{CF}$.

\section{Methods}

Participants

Children participating in the Australian Respiratory Early Surveillance Team for CF (AREST CF) programme at Princess Margaret Hospital in Perth, undergoing chest computed tomography (CT) and BAL as part of their annual review, were asked to participate in this study. The programme includes a bronchoscopy with BAL and a chest CT scan performed at 3 months of age and then annually until the age of 6 years, as previously described [19]. All patients in this study had OPS performed at the time of the CT and BAL, thus paired upper (OPS) and lower (BAL) airway cultures were collected at each visit for each participating patient. All patients that had paired OPS-BAL performed were included in the study. Children were clinically stable at the time of assessment. Ethics approval for the research programme was obtained from the hospital ethics committee. Written, informed consent was obtained from parents prior to each annual review.

Clinical practice at the hospital is to attempt eradication of $P$. aeruginosa when detected on BAL, with 2 weeks of intravenous antibiotics followed by 4 weeks of inhaled tobramycin and oral ciprofloxacin in young patients [20]. A bronchoscopy is performed after completing the eradication protocol for patients with a first lower airway $P$. aeruginosa infection and according to clinical judgement for patients with repeated $P$. aeruginosa infection. A successful eradication rate of $77 \%$ was previously shown for this protocol [20].

Positive OPS culture is not routinely treated if $P$. aeruginosa was not simultaneously detected on BAL. OPS cultures are not collected at routine clinic visits. Routine therapy for mild respiratory exacerbations, at our centre, often includes inhaled or oral antibiotics targeting P. aeruginosa, regardless of previous BAL or OPS culture results.

\section{Sampling procedure}

Following induction of general anaesthesia, OPS was performed and sent for culture of $P$. aeruginosa only. Children were then intubated and a chest CT scan performed using a low-dose scanning protocol with pressure-controlled inspiratory and expiratory series [7]. The endotracheal tube was replaced by a laryngeal mask airway for flexible bronchoscopy, and BAL was performed 1) in the right middle lobe and 2) in the worst affected lobe as identified by the preceding chest CT or the lingula if the right middle lobe was the worst affected. Topical lignocaine to the vocal cords or trachea was withheld until after all microbiological samples were obtained. Suction of pulmonary secretions was delayed until the tip of the bronchoscope was below the level of the carina to avoid upper airway contamination. The first aliquot from each lobe was sent for standard microbiological processing for the existence of bacteria, fungi and viruses. For $P$. aeruginosa, the criterion for infection was the presence of the organism at any density in BAL or at any density in OPS cultures, as previously described [21].

\section{Inflammation}

To determine inflammation levels, free neutrophil elastase activity and interleukin (IL)-8 concentration were measured, as previously described $[19,22]$. The lower limit of detection of free neutrophil elastase 
activity was $100 \mathrm{ng} \cdot \mathrm{mL}^{-1}$ and visits were defined as neutrophil elastase-positive if the concentrations were above the lower limit of detection.

\section{CT analysis}

The PRAGMA-CF (Perth-Rotterdam Annotated Grid Morphometric Analysis for CF) analysis method was used to determine the extent of structural lung disease. The overall extent of lung disease (\%Disease) and bronchiectasis (\%Bronchiectasis) was determined from inspiratory scans, and the extent of trapped air (\%TrappedAir) was determined from the expiratory scan, as previously described [23]. Each CT scan used in this study was scored by only one individual. A high interobserver and intraobserver correlation for this scoring system was previously shown [23]. The person scoring the scans was unaware of OPS or BAL results.

\section{Hospitalisation}

Information regarding hospitalisation, between January 2005 and October 2017, in the 12 months following sampling was extracted from hospital records. Included for analysis were only hospitalisations for i.v. treatment of acute respiratory symptoms (respiratory exacerbations). Hospitalisations within 1 month of the routine bronchoscopy were excluded from analysis in order to avoid including admissions due to bronchoscopy and BAL findings alone (e.g. finding excessive thick airway secretions), which may include children who did not have respiratory symptoms reported at the time.

\section{Statistical analysis}

Data were summarised by standard descriptive statistics. Culture results for P. aeruginosa from the BAL samples (lower airway culture) were considered to be "gold standard" for analysis and used to determine the ability of OPS cultures (upper airway culture) to reflect current infection. Sensitivity, specificity, positive predictive value, negative predictive value and area under the receiver operating characteristic (ROC) curve were calculated using generalised estimating equation (GEE) analysis, taking into account repeated measures in the same patients. Pre- and post-test probabilities were presented using Fagan's nomogram and Cohen's $\kappa$ was used for concordance analysis. In addition, the ability of BAL and OPS to predict BAL culture positivity in the following annual review was assessed. Cross-sectional and longitudinal analyses were performed comparing OPS and BAL results at baseline with inflammation markers and CT outcomes in the same year and at next annual review, respectively. Mixed effects models were used for continuous outcomes and GEE models for dichotomous outcomes, clustering for repeated visits in the same patients, and correcting for age and pancreatic sufficiency. Longitudinal analyses were additionally adjusted for inflammatory or CT outcomes at baseline. Clustered negative binomial models were used to estimate incidence rate ratios (IRRs) for hospitalisations for respiratory exacerbations comparing children with positive and negative culture results, for both OPSs and BAL. All data were analysed using Stata version 14 (StataCorp, College Station, TX, USA) and graphs were produced using Prism version 6 (GraphPad, La Jolla, CA, USA).

\section{Results}

181 patients participated in this study between March 2005 and April 2017. These patients underwent 690 annual visits in which paired upper (OPS) and lower (BAL) airway cultures were obtained. Patients' characteristics are presented in table 1. Growth of P. aeruginosa was found in 10.1\% (70 out of 690) of the upper airway (OPS) cultures and in 7.4\% (51 out of 690) of the lower airway (BAL) cultures. During the study period 35 (19.3\%) patients had at least one lower airway infection with $P$. aeruginosa.

$\begin{array}{lc}\text { TABLE } 1 \text { Study population characteristics at the annual reviews } & \\ \text { Participants } & 181 \\ \text { Male } & 99(55) \\ \text { Pancreatic sufficiency } & 27(15) \\ \text { Total annual visits (with paired OPS-BAL) } & 690 \\ \text { Visits per patient } & 3.8(1 ; 8) \\ \text { Age of patient at visit years } & 3.02(1.24-4.91) \\ \text { Visits with BAL inflammatory markers } & 668 \\ \text { Visits with CT scans (PRAGMA-CF) } & 550\end{array}$

Data are presented as $\mathrm{n}, \mathrm{n}$ (\%), median (minimum; maximum) or median (interquartile range). OPS: oropharyngeal swab; BAL: bronchoalveolar lavage; CT: computed tomography; PRAGMA-CF: PerthRotterdam Annotated Grid Morphometric Analysis for Cystic Fibrosis. 
TABLE 2 Prevalence of organisms, diagnostic accuracy and likelihood ratios of oropharyngeal swabs (OPSs) compared with bronchoalveolar lavage (BAL) for detection of Pseudomonas aeruginosa

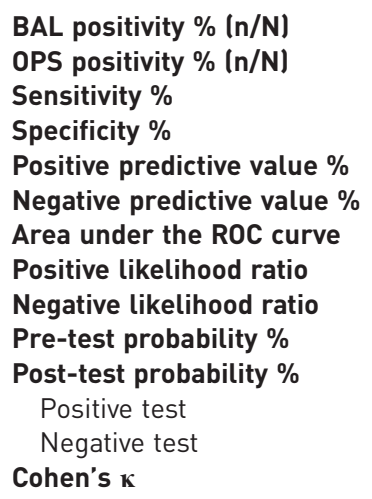

$7.4(51 / 690)$

$10.1(70 / 690)$

$23.0(13.5-36.4)$

$91.4(88.3-93.7)$

$18.2(10.4-29.8)$

$93.7(90.6-95.8)$

$0.57(0.51-0.63)$

$2.59(1.49-4.5)$

$0.84(0.72-0.98)$

$18.2(10.4-29.8)$

$6.3(4.2-9.4)$

Data presented with $95 \%$ confidence intervals, unless otherwise stated. All calculations performed using generalised estimating equation models to correct for correlated measurements in the same individuals. ROC: receiver operating characteristic.

\section{Oropharyngeal cultures as surrogates for lower airway infection}

The prevalence of $P$. aeruginosa in upper and lower airway cultures, and the sensitivity, specificity, positive predictive value, negative predictive value and area under the ROC curve for upper airway culture as a surrogate for current lower airway culture, are provided in table 2. The likelihood ratios and Fagan's nomogram demonstrate that a negative OPS culture marginally decreases the probability for a positive lower airway (BAL) culture from a pre-test probability of $7.4 \%$ to a post-test probability of $6.3 \%$. Concordance analysis shows a poor agreement between the two tests with a Cohen's $\kappa$ of 0.123 . The ROC curve is provided in figure 1.

\section{Disease severity at time of assessment}

When evaluating disease severity as assessed by inflammatory and structural lung disease present at the time of the annual visit, a positive $P$. aeruginosa culture on OPS did not have a significant association with any markers of inflammation and had borderline significant association to only one of the PRAGMA CT scores (\%Disease; $\mathrm{p}=0.054$ before adjusting for age and pancreatic sufficiency, and $\mathrm{p}=0.043$ after adjustment) (table $3)$; this association was lost after adjusting for concurrent BAL results $(\mathrm{p}=0.11)$. In contrast, a positive $P$. aeruginosa culture on BAL was significantly associated with the presence of neutrophil elastase on BAL, elevated IL-8 levels on BAL and an increase in all structural lung disease scores as measured by PRAGMA CT (\%Disease, \%Bronchiectasis and \%TrappedAir; $\mathrm{p}<0.001$ ) (table 3 and figure 2 ).

\section{Impact on future disease and future lower airway P. aeruginosa infection}

OPS results, BAL and CT data for the next year were available for 498 time-points in 181 patients. When evaluating future disease severity as assessed by inflammatory and structural lung disease 1 year later, a current positive $P$. aeruginosa culture on OPS did not have any significant association with any future

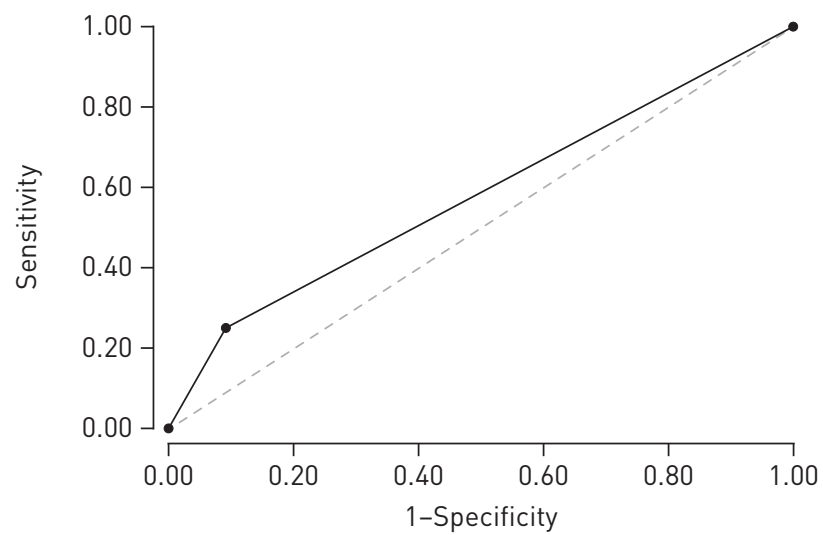

FIGURE 1 Receiver operator characteristic curve for oropharyngeal swabs to predict lower airway (bronchoalveolar lavage) infection with Pseudomonas aeruginosa. 
TABLE 3 Association of bronchoalveolar lavage (BAL) and oropharyngeal swab (OPS) infection status with inflammatory markers and PRAGMA (Perth-Rotterdam Annotated Grid Morphometric Analysis) computed tomography (CT) score in the same year

BAL culture (positive versus negative)

OPS culture (positive versus negative)

n $(95 \% \mathrm{Cl})$

p-value

n $(95 \% \mathrm{Cl})$

p-value

Inflammatory markers ${ }^{\#}$
Neutrophil elastase
Interleukin-8 levels (log scale)
PRAGMA CT scores ${ }^{\text {I }}$
\%Disease
\%Bronchiectasis
\%TrappedAir

$\begin{array}{cr}\text { OR } 4.17(2.04-8.53) & <0.001 \\ \text { Difference } 0.92(0.55-1.29) & <0.001 \\ & \\ 1.56(0.96-2.16) & <0.001 \\ 0.57(0.24-0.90) & 0.001 \\ 4.12(2.05-3.19) & <0.001\end{array}$

OR $1.17(-0.58-2.40)$
Difference $0.26(-0.07-0.59)$
$0.55(0.02-1.08)$
$0.08(-0.20-0.37)$
$0.66(-1.16-2.18)$

0.658

0.124

0.043

0.561

0.479

Mixed models for continuous outcomes; generalised estimating equation models for dichotomous outcome (i.e. neutrophil elastase yes/no). Analyses clustered for repeated measures in same patient, and adjusted for age at visit and pancreatic sufficiency. ${ }^{\#}: \mathrm{n}=668 ;{ }^{\uparrow}: \mathrm{n}=550$.

markers of inflammation, PRAGMA CT scores or change in PRAGMA CT scores (table 4). Conversely, a current positive $P$. aeruginosa culture on BAL was significantly associated with worse future structural lung disease, as assessed on PRAGMA CT scores (\%Disease and \%Bronchiectasis; p $<0.05$ ). Furthermore, adjusting for prior year CT scores showed that in patients with $P$. aeruginosa culture on BAL there was a significant progression in structural lung disease 1 year later (\%Disease and \%Bronchiectasis on PRAGMA CT scores; $\mathrm{p}<0.05)$ (table 4).

The prevalence of lower airway (BAL) P. aeruginosa 1 year later was $7.6 \%$ (38 out of 498). Patients with $P$. aeruginosa culture on BAL but not on OPS had an increased risk for positive $P$. aeruginosa on BAL in the next annual review (OR 5.18, 95\% CI 2.22-12.07; $\mathrm{p}<0.001$ versus OR 2.03, 95\% CI $0.85-4.87 ; \mathrm{p}=0.11$, respectively). Importantly, as mentioned earlier, the practice in our hospital is to treat $P$. aeruginosa when detected on BAL; positive OPS cultures performed at the time of BAL are not routinely treated.

\section{Hospitalisations for respiratory exacerbations}

Data on hospitalisations for respiratory exacerbations are presented in table 4. 219 hospital admissions were recorded in participating patients during the study period. Excluded from risk analysis were 51
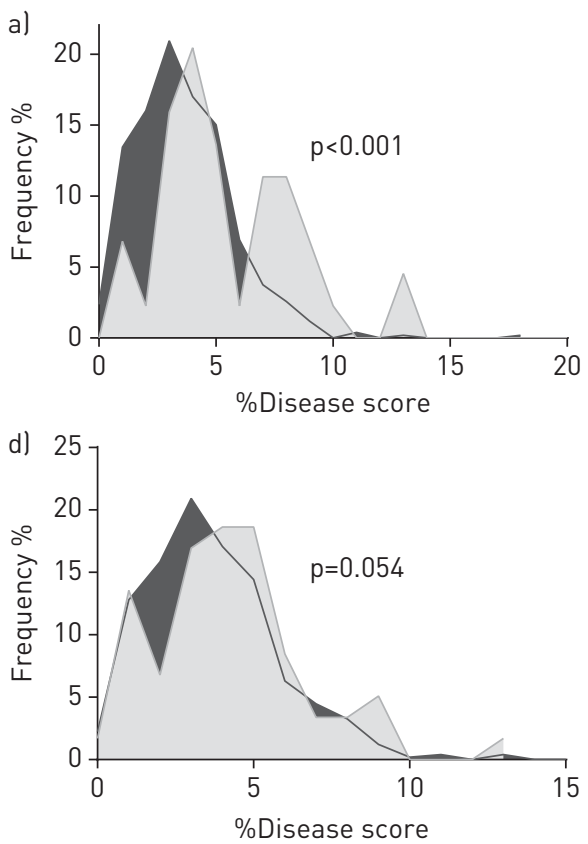
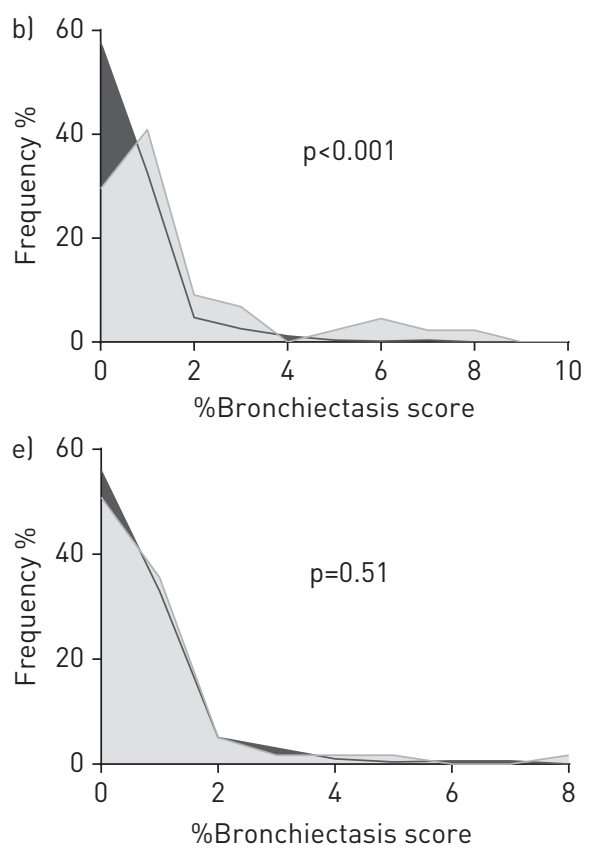
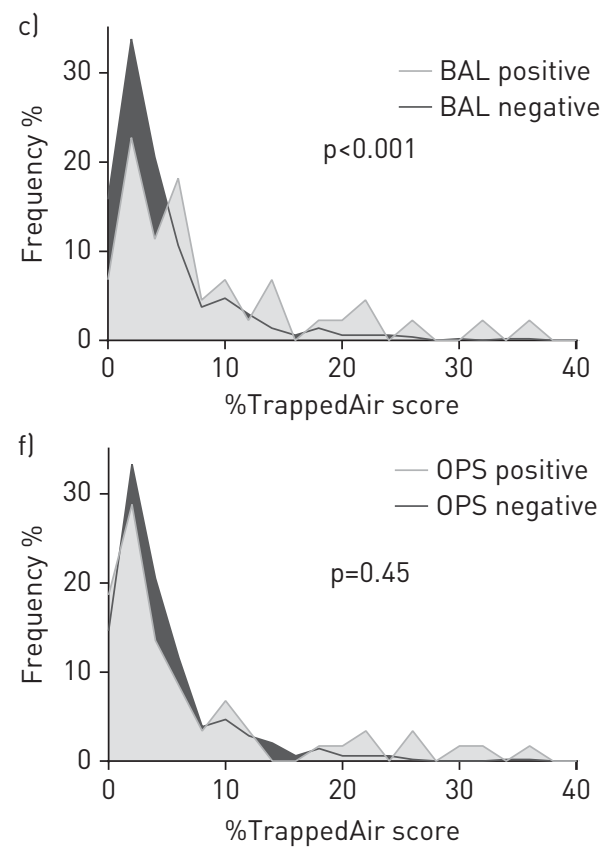

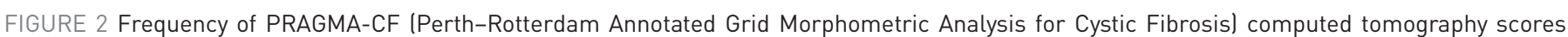

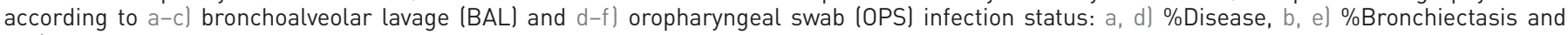
c, f) \%TrappedAir. 
TABLE 4 Association of bronchoalveolar lavage (BAL) and oropharyngeal swab (OPS) infection status with inflammatory markers, PRAGMA (Perth-Rotterdam Annotated Grid Morphometric Analysis) computed tomography (CT) and admission rates for respiratory exacerbations in the following year

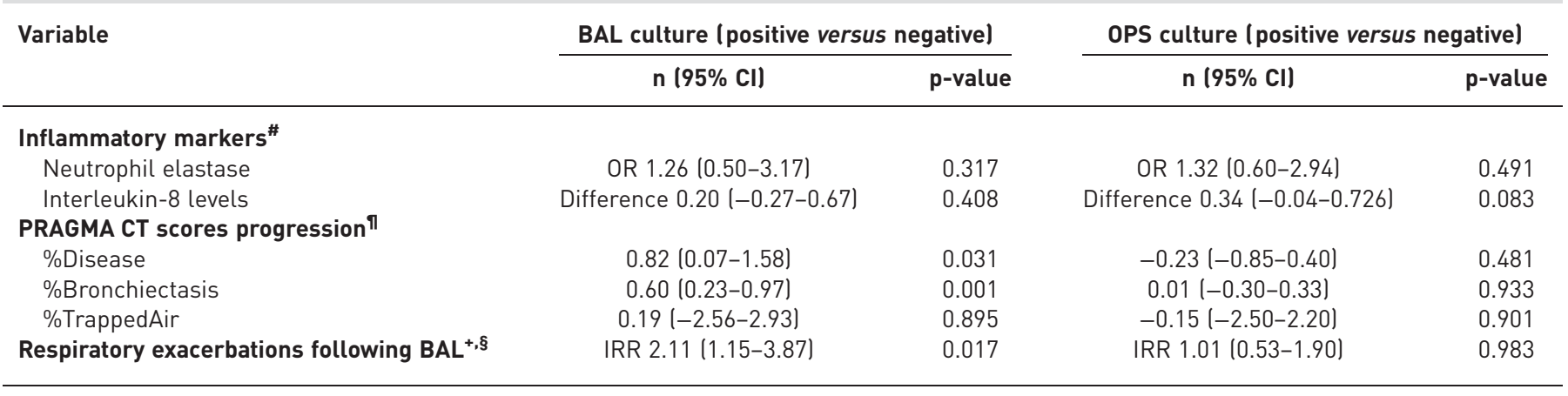

IRR: incidence rate ratio. Mixed models for continuous outcomes; generalised estimating equation models for dichotomous outcome li.e. neutrophil elastase); negative binomial model for count (number of admissions). All analyses clustered for repeated measures in same patient, adjusted for age at visit and pancreatic sufficiency. Analyses on inflammatory markers and CT scores in following year adjusted for respective results at time of airway sampling. ${ }^{\#}: n=470 ;{ }^{\natural}: n=369 ;{ }^{+}: n=690 ;{ }^{\S}:$ in 12 months following BAL, excluding admissions in first month after BAL.

admissions for eradication of $P$. aeruginosa detected on BAL, two admissions for eradication of meticillin-resistant Staphylococcus aureus detected on BAL and another 27 admissions that occurred in close proximity (within 1 month) to the bronchoscopy. 139 admissions were included in the risk analysis.

Importantly, following the 51 admissions for eradication of $P$. aeruginosa a "post-eradication regimen" bronchoscopy was performed on 30 occasions with a successful eradication documented in 28 (93\%).

Patients with positive $P$. aeruginosa infection on an OPS were not at an increased risk for hospitalisations for respiratory exacerbation in the year following culture when compared with patients with a negative OPS (IRR 1.03, 95\% CI 0.54-1.97; p=0.91). In contrast, the IRR for hospitalisation for subjects with positive versus negative BAL was 2.09 (95\% CI 1.14-3.81; $\mathrm{p}=0.016)$. As these results were obtained after exclusion of admissions for Pseudomonas eradications and early post-bronchoscopy admissions (as described earlier) they might even underestimate the risk for hospital admission in lower airway $P$. aeruginosa infection.

\section{Discussion}

In this large observational study of prospectively collected data in young children with CF, OPS cultures for $P$. aeruginosa were poorly concordant to BAL culture results and were not associated with markers of lung disease severity. Neither positive nor negative OPS cultures were correlated to lower airway inflammation or progression of structural disease, or associated with hospitalisations for respiratory exacerbations or predicted future lower airway infection with $P$. aeruginosa, despite the fact that no routine treatment was directed against positive OPSs in asymptomatic patients. In contrast, $P$. aeruginosa infection on BAL was associated with lower airway inflammation, current and progression of structural lung disease, and with an increased risk for respiratory exacerbations and future lower airway P. aeruginosa infection. Our results, from a well-characterised, unselected cohort, provide objective and definitive data with which to assess the clinical utility of upper airway bacterial surveillance in young children with $\mathrm{CF}$.

Previous studies assessing the significance of upper airway culture results in children with CF have shown, very similar to our results, that OPSs underrepresent bacteria associated with airway inflammation when compared with lower airway cultures and that in young children with $\mathrm{CF}$ increased endobronchial inflammation is associated with $P$. aeruginosa infection on BAL and not with upper airway $P$. aeruginosa-positive cultures $[3,4]$. To the best of our knowledge, no prior study has evaluated the relation between upper airway culture results and clinic parameters such as presence and progression of structural lung disease on chest CT scans, respiratory symptoms or risk for respiratory exacerbations. In contrast, the presence of pathogenic organisms in the lower airways, especially $P$. aeruginosa, in children with CF has been repeatedly shown to be associated with worse clinical outcomes [1-8].

Current guidelines recommend the use of oropharyngeal cultures for bacterial surveillance in young children with CF $[9,10]$ despite stating that oropharyngeal cultures do not reliably predict the presence of pathogens in lower airways $[9,24,25]$. Current practice to routinely evaluate OPSs and consider treatment according to culture result is largely based on the findings that OPS cultures have high specificity and negative predictive values for the detection of lower airway $P$. aeruginosa infection [12-15]. Consequently, 
several reports have suggested that OPS cultures can be used to rule out $P$. aeruginosa infection, with a negative predictive value of $>90 \%[9,14]$. Importantly, negative predictive value is heavily dependent on disease prevalence. In our cohort $7.4 \%$ of children had P. aeruginosa in BAL, similar to previous reports $(8-11 \%)$ in young children $[12,14]$, implying that the negative predictive value cannot be $<92.6 \%$, regardless of the test characteristics. In order to rule out $P$. aeruginosa with certainty OPSs would need to have a very high sensitivity and, consequently, very low rates of false negatives. Our study confirms previous reports that the sensitivity of OPSs is relatively low. As a result of this a negative OPS result reduced the probability for a positive BAL only marginally from $7.4 \%$ (pre-test) to $6.3 \%$ (post-test). This is unlikely to be associated with any meaningful clinical consequences, and explains the lack of significant associations between OPS results and the tested clinical outcomes.

The lack of correlation between OPS results and clinical outcomes and marginal effect on ruling out a lower airway $P$. aeruginosa infection brings to question the safety and efficiency of treating patients according to their OPS culture results. Using OPS results to guide treatment may result in administration of unnecessary or in delayed therapy, i.e. in our cohort 83\% (58 out of 70) of patients with a positive OPS would be receiving unnecessary treatment (due to a false-positive result) and $76 \%$ (39 out of 51) of patients with a lower airway $P$. aeruginosa infection would not be receiving appropriated timely treatment (due to a false-negative result). This carries both the risk of adverse drug reactions in healthy individuals as well as failing to prevent the progression of lung disease by early initiation of treatment in patients.

Indeed, routine use of OPSs is a matter of ongoing debate in asymptomatic young patients and many have stressed that an alternative method should be considered for early detection of lower airway P. aeruginosa infection in order to prevent progression of lung disease $[4,12,26]$. This is especially true in view of our results that show worse clinical outcomes in children with $P$. aeruginosa BAL infection, demonstrating that annual surveillance with BAL is not sufficient to prevent disease progression. However, the progression in structural lung disease seen in patients with $P$. aeruginosa BAL infection may also be related to ongoing inflammation.

Currently, alternative methods used in clinical practice include oropharyngeal suctioning after cough stimulation or after sputum induction with hypertonic saline. Both methods were recently compared with BAL: the cough stimulation technique showed high specificity and slightly better sensitivity than OPSs [27], and the sputum induction technique showed variable results with good bacteriological correlation in a small study [28], but low specificity, sensitivity and predictive values in a larger study [21]. Use of molecular diagnostic techniques on routine clinical samples holds the promise of improved accuracy in the diagnosis of infection. Molecular techniques, such as PCR, have high sensitivity for detection of $P$. aeruginosa in expectorated sputum, but no study has yet evaluated the use of PCR on BAL fluid in young nonexpectorating children with CF. Further research is warranted to investigate if molecular techniques applied to upper airway samples may provide improved accuracy in early detection of lower respiratory tract pathogens in young asymptomatic patients with CF [16]. A promising technique is the analysis of compounds in exhaled breath. Hydrogen cyanide in exhaled breath has been shown to be a specific marker of $P$. aeruginosa infection in children and adults [29-31]. Gas chromatography-mass spectrometry techniques analysing volatile organic compounds in exhaled breath may, in the future, also improve the sensitivity for detection of $P$. aeruginosa and allow real-time infection risk assessment, thereby facilitating appropriate clinical decision making [32, 33].

The study has several limitations. First, much like previous studies comparing OPSs with BAL [12-14], samples were obtained in anaesthetised children, which has raised concerns that samples do not sufficiently reflect OPSs obtained in routine clinical practice. Still, we have identified more P. aeruginosa in OPS than BAL cultures and predictive values of our results match with previous reported studies, demonstrating that the culture technique is at least adequate. Second, OPSs were sampled in stable individuals during a routine annual review and therefore our results may not be fully generalised to the clinical significance of OPSs in symptomatic young patients with CF. Furthermore, OPSs are not routinely collected in our centre at each visit, thus we have no data on the value of frequent repeated upper airway sampling, which has been shown to reduce the number of false-negative cultures [15]. Performing repeated OPSs at quarterly intervals might have allowed a better evaluation of the temporal association between upper and lower airway infection. Third, molecular typing of the P. aeruginosa strains isolated in OPS and BAL cultures was not available in the current study. Molecular typing may have assisted in accurately characterising the association between current and future upper and lower airway infections. Notably, previous studies assessing this association have shown a molecular discordance between BAL and oropharyngeal $P$. aeruginosa strains $[12,34]$.

The main strength of our study lies in the large number of included patients with annually repeated paired OPS-BAL cultures and the longitudinal design. This is the largest study to date comparing results from 
paired upper and lower airway cultures in young clinically stable children with CF, and the longitudinal design enables assessment of the clinical significance of the culture results.

In summary, OPS Pseudomonas culture results only slightly reduce the probability for a negative lower airway infection and are not associated with parameters of lower airway disease in young clinically stable patients with CF. These findings do not support the routine use of OPS cultures in ruling out lower airway infections, and highlight the need to develop a more robust feasible method for early detection of lower airway $P$. aeruginosa infection that will help guide treatment and prevent or delay the progression of destructive lung disease in young children.

Acknowledgements: The full authorship includes the members of AREST CF. The full list of AREST CF members can be found at www.arestcf.org.

Conflict of interest: None declared.

Support statement: The AREST CF early surveillance programme is funded by grants from the National Health and Medical Research Council (NHMRC) Australia, the National Institutes of Health, Cystic Fibrosis Foundation Therapeutics Inc. and the University of Western Australia. O. Breuer has been supported by a Lowy Foundation Paediatric Fellowship arranged by AUSiMED (Australia/Israel Medical Research). D. Caudri received support for a Research Fellowship from the Rothwell Foundation, the Ter Meulen Grant of the Royal Netherlands Academy of Arts and Sciences, and the Sophia Childrens' Hospital Fund. AREST CF is supported by Cystic Fibrosis Foundation Therapeutics Inc. and CF Australia, and NHMRC grants APP1000896 and 1020555 . None of the funding bodies were in any way involved in the data collection, interpretation of the data or writing of the manuscript.

\section{References}

1 Gangell C, Gard S, Douglas T, et al. Inflammatory responses to individual microorganisms in the lungs of children with cystic fibrosis. Clin Infect Dis 2011; 53: 425-432.

2 Sly PD, Gangell CL, Chen L, et al. Risk factors for bronchiectasis in children with cystic fibrosis. N Engl J Med 2013; 368: 1963-1970.

3 Zemanick ET, Wagner BD, Robertson CE, et al. Assessment of airway microbiota and inflammation in cystic fibrosis using multiple sampling methods. Ann Am Thorac Soc 2015; 12: 221-229.

4 Sagel SD, Gibson RL, Emerson J, et al. Impact of Pseudomonas and Staphylococcus infection on inflammation and clinical status in young children with cystic fibrosis. J Pediatr 2009; 154: 183-188.

5 Armstrong DS, Grimwood K, Carlin JB, et al. Lower airway inflammation in infants and young children with cystic fibrosis. Am J Respir Crit Care Med 1997; 156: 1197-1204.

6 Ramsey KA, Ranganathan S, Park J, et al. Early respiratory infection is associated with reduced spirometry in children with cystic fibrosis. Am J Respir Crit Care Med 2014; 190: 1111-1116.

7 Mott LS, Park J, Murray CP, et al. Progression of early structural lung disease in young children with cystic fibrosis assessed using CT. Thorax 2012; 67: 509-516.

8 Stick SM, Brennan S, Murray C, et al. Bronchiectasis in infants and preschool children diagnosed with cystic fibrosis after newborn screening. J Pediatr 2009; 155: 623-628.

9 Borowitz D, Robinson KA, Rosenfeld $\mathrm{M}$, et al. Cystic Fibrosis Foundation evidence-based guidelines for management of infants with cystic fibrosis. J Pediatr 2009; 155: 6 Suppl., S73-S93.

10 Smyth AR, Bell SC, Bojcin S, et al. European Cystic Fibrosis Society Standards of Care: Best Practice guidelines. J Cyst Fibros 2014; 13: Suppl. 1, S23-S42.

11 Lahiri T, Hempstead SE, Brady C, et al. Clinical practice guidelines from the cystic fibrosis foundation for preschoolers with cystic fibrosis. Pediatrics 2016; 137: e20151784.

12 Armstrong DS, Grimwood K, Carlin JB, et al. Bronchoalveolar lavage or oropharyngeal cultures to identify lower respiratory pathogens in infants with cystic fibrosis. Pediatr Pulmonol 1996; 21: 267-275.

13 Ramsey BW, Wentz KR, Smith AL, et al. Predictive value of oropharyngeal cultures for identifying lower airway bacteria in cystic fibrosis patients. Am Rev Respir Dis 1991; 144: 331-337.

14 Rosenfeld M, Emerson J, Accurso F, et al. Diagnostic accuracy of oropharyngeal cultures in infants and young children with cystic fibrosis. Pediatr Pulmonol 1999; 28: 321-328.

15 Burns JL, Gibson RL, McNamara S, et al. Longitudinal assessment of Pseudomonas aeruginosa in young children with cystic fibrosis. J Infect Dis 2001; 183: 444-452.

16 Tramper-Stranders GA, van der Ent CK, Wolfs TF. Detection of Pseudomonas aeruginosa in patients with cystic fibrosis. J Cyst Fibros 2005; 4: Suppl. 2, 37-43.

17 Dijk FN, McKay K, Barzi F, et al. Improved survival in cystic fibrosis patients diagnosed by newborn screening compared to a historical cohort from the same centre. Arch Dis Child 2011; 96: 1118-1123.

18 Frederiksen B, Koch C, Hoiby N. Changing epidemiology of Pseudomonas aeruginosa infection in Danish cystic fibrosis patients (1974-1995). Pediatr Pulmonol 1999; 28: 159-166.

19 Sly PD, Brennan S, Gangell C, et al. Lung disease at diagnosis in infants with cystic fibrosis detected by newborn screening. Am J Respir Crit Care Med 2009; 180: 146-152.

20 Douglas TA, Brennan S, Gard S, et al. Acquisition and eradication of $P$. aeruginosa in young children with cystic fibrosis. Eur Respir J 2009; 33: 305-311.

21 D'Sylva P, Caudri D, Shaw N, et al. Induced sputum to detect lung pathogens in young children with cystic fibrosis. Pediatr Pulmonol 2017; 52: 182-189.

22 Berry LJ, Sheil B, Garratt L, et al. Stability of interleukin 8 and neutrophil elastase in bronchoalveolar lavage fluid following long-term storage. J Cyst Fibros 2010; 9: 346-350.

23 Rosenow T, Oudraad MC, Murray CP, et al. PRAGMA-CF. A quantitative structural lung disease computed tomography outcome in young children with cystic fibrosis. Am J Respir Crit Care Med 2015; 191: 1158-1165. 
24 Robinson KA, Saldanha IJ, McKoy NA. Management of infants with cystic fibrosis: a summary of the evidence for the cystic fibrosis foundation working group on care of infants with cystic fibrosis. J Pediatr 2009; 155: 6 Suppl., S94-S105.

25 Mogayzel PJ Jr, Naureckas ET, Robinson KA, et al. Cystic Fibrosis Foundation pulmonary guideline. pharmacologic approaches to prevention and eradication of initial Pseudomonas aeruginosa infection. Ann Am Thorac Soc 2014; 11: $1640-1650$

26 Furness JC, Habeb A, Spencer DA, et al. To the editor: Bronchoalveolar lavage (BAL) in pediatric cystic fibrosis (CF): its clinical use modified by audit in a regional CF center. Pediatr Pulmonol 2002; 33: 234.

27 Doumit M, Belessis Y, Stelzer-Braid S, et al. Diagnostic accuracy and distress associated with oropharyngeal suction in cystic fibrosis. J Cyst Fibros 2016; 15: 473-478.

28 Blau $\mathrm{H}$, Linnane $\mathrm{B}$, Carzino $\mathrm{R}$, et al. Induced sputum compared to bronchoalveolar lavage in young, non-expectorating cystic fibrosis children. J Cyst Fibros 2014; 13: 106-110.

29 Gilchrist FJ, Belcher J, Jones AM, et al. Exhaled breath hydrogen cyanide as a marker of early Pseudomonas aeruginosa infection in children with cystic fibrosis. ERJ Open Res 2015; 1: 00044-2015.

30 Gilchrist FJ, Bright-Thomas RJ, Jones AM, et al. Hydrogen cyanide concentrations in the breath of adult cystic fibrosis patients with and without Pseudomonas aeruginosa infection. J Breath Res 2013; 7: 026010.

31 Enderby B, Smith D, Carroll W, et al. Hydrogen cyanide as a biomarker for Pseudomonas aeruginosa in the breath of children with cystic fibrosis. Pediatr Pulmonol 2009; 44: 142-147.

32 Martinez-Lozano Sinues P, Zenobi R, Kohler M. Analysis of the exhalome: a diagnostic tool of the future. Chest 2013; 144: 746-749.

33 van Mastrigt E, Reyes-Reyes A, Brand $\mathrm{K}$, et al. Exhaled breath profiling using broadband quantum cascade laser-based spectroscopy in healthy children and children with asthma and cystic fibrosis. J Breath Res 2016; 10: 026003.

34 Kidd TJ, Ramsay KA, Vidmar S, et al. Pseudomonas aeruginosa genotypes acquired by children with cystic fibrosis by age 5-years. J Cyst Fibros 2015; 14: 361-369. 